\title{
Calcineurin inhibitors in the treatment of allergic dermatitis
}

\author{
Ana Paula Beltran Moschione Castro*
}

\begin{abstract}
Objective: To review the role of calcineurin inhibitors in the treatment of allergic dermatitis, focusing on mechanisms of action, efficacy and topical and systemic adverse effects.

Sources: Articles written in English and published in MEDLINE using the following keywords: pimecrolimus, tacrolimus, calcineurin inhibitors. Original articles that presented controlled and open studies for assessing efficacy, tolerability and adverse effects were selected. Review articles and case series were also evaluated; the latter was only considered for assessing adverse effects. The official websites of the Food and Drug Administration and of manufacturers of calcineurin inhibitors were also used.

Summary of the findings: The data showed that calcineurin inhibitors are efficient in the treatment of mild to severe atopic dermatitis, leading to improvement in symptoms, reduction in number of attacks and need of topical corticotherapy. Calcineurin inhibitors have good tolerability and few topical adverse effects. To date, there has been no evidence to support higher prevalence of neoplasia in patients using these drugs; however, an adequate pharmacovigilance system has been set up to assess this aspect.

Conclusions: Calcineurin inhibitors, which are a new drug class in the treatment of allergic dermatitis, are efficient, well tolerated and have few adverse effects. Calcineurin inhibitors should always be used according to recommended guidelines, and patients should always be followed by the physician during and after their administration.
\end{abstract}

J Pediatr (Rio J). 2006;82(5 Suppl):S166-72: Pimecrolimus, tacrolimus, calcineurin inhibitors.

\section{Introduction}

Dermatologic diseases are among the most frequent clinical manifestations in pediatric patients. Chronic and recurrent allergic conditions pose a real challenge to daily medical practice. Atopic dermatitis is probably the best example. It has an early onset, usually by 5 years of age, stands out as the most frequent chronic skin disease in children and can affect up to $20 \%$ of this population. Atopic dermatitis has a multifactorial etiology, comprehending the individual's genetic characteristics that result in immunological and skin barrier changes. Such changes are also associated with environmental factors, such as infectious agents, food allergens and aeroallergens. Atopic dermatitis $(A D)$ is strongly associated with other allergic

* Mestre. Médica assistente e responsável, Ambulatório de Dermatite Atópica, Unidade de Alergia e Imunologia, Instituto da Criança, Hospital de Clínicas, Faculdade de Medicina, Universidade de São Paulo (USP), São Paulo, SP, Brasil.

Suggested citation: Castro AP. Calcineurin inhibitors in the treatment of allergic dermatitis. J Pediatr (Rio J). 2006;82(5 Suppl):S166-72. manifestations, such as asthma and rhinitis, which reveals its inflammatory nature combined with immunological impairment. ${ }^{1,2}$

The clinical signs and symptoms presented by patients result from the complex interaction of skin cells, such as keratinocytes and Langerhans cells, with immune system cells, especially mast cells, lymphocytes and eosinophils. There are increased IgE levels and a defined profile of allergic disease in around $80 \%$ of $A D$ in children. These patients have enhanced synthesis of interleukin (IL) related to atopy, such as IL-4, IL-5 and IL-13. Activation of mast cells and release of their mediators, especially histamine, tryptase and leukotrienes, lead to vasodilatation and pruritus, which induces scratching. Keratinocytes synthesize IL and express adhesion molecules that increase the inflammatory process. Both subpopulations of dendritic cells in the skin, Langerhans cells and dendritic cells in the epithelium, called inflammatory dendritic epidermal cells (IDEC), have high-affinity IgE receptors in their surface and facilitate the activation of T lymphocytes. ${ }^{3,4}$ 
Infectious agents, such as Staphylococcus aureus, produce toxins that act as superantigens and intensely stimulate lymphocytes and subsequent production of IL. Food allergens and aeroallergens provide degranulation of mast cells via IgE-mediated mechanism. These changes culminate in intense inflammatory process and tissue lesion. Controlling dermatitis comprehends several management strategies; however, all patients require measures to repair the skin barrier and control inflammation. 2,3

Contact dermatitis is another skin disease characterized by changes in local immunity. Nevertheless, the process starts after sensitivity to contact allergen or to a substance acting as hapten. Haptens are substances that do not induce immunologic response independently; however, when associated with protein in the organism, haptens form a complex that can be presented to the immune system and induce inflammatory response. In allergic contact dermatitis, topical drugs, cosmetics, resins, among others, may cause skin lesions. In this case, there is an infiltrate of lymphocytes that synthesize IL and cause tissue lesion. Although the prevalence of contact dermatitis in several pediatric age groups is not known, there has been an increase in this population, especially due to early use of a series of substances, such as enamels, cosmetics, hair dyes and other products that may cause sensitivity and skin eczema. ${ }^{5}$

\section{Calcineurin inhibitors}

Topical calcineurin inhibitors have originated from the need of using drugs to efficiently control the inflammatory process and be well tolerated without causing adverse effects, even in the long term.

Calcineurin is a cytoplasmatic protein present in several cells, including lymphocytes and dendritic cells. After activation, it acts as a transcription factor of inflammatory IL, such as IL-2, IL-3, IL-4 and TNF-alpha. Such activation is a calcium-dependent mechanism that also includes two types of proteins: calmodulin and immunophilins. The latter were given that name because they act as receptors of calcineurin-inhibiting substances. (Figure 1 ). ${ }^{6}$

Inhibiting calcineurin means to minimize lymphocyte activity; logically, this process should be performed in a completely controlled manner to assure improvement in the allergic condition without compromising the immune function of the organism. The drugs able to reduce the action of calcineurin act by inhibiting different immunophilins. There are systemic calcineurin inhibitors, such as, for example, cyclosporine. However, the challenge was to develop efficacious drugs for topical use; thus, systemic adverse effects could be minimized or absent. Calcineurin inhibitors, such as cyclosporine, have pharmacological characteristics that avoid achieving the desired efficacy in their topical use. ${ }^{6}$ There are two calcineurin inhibitors available for topical use: pimecrolimus and tacrolimus.

\section{Pimecrolimus}

Macrolides with immunosuppressive properties were discovered in the 1980's. Since then, they have been used in diseases where the main damage is related to immune system dysfunction, i.e., self-immune diseases, or to control rejection of transplants. Pimecrolimus was synthesized during the development process of this class of drugs. It is a macrolide originated from ascomycin, one of the lactam substances produced by Streptomyces
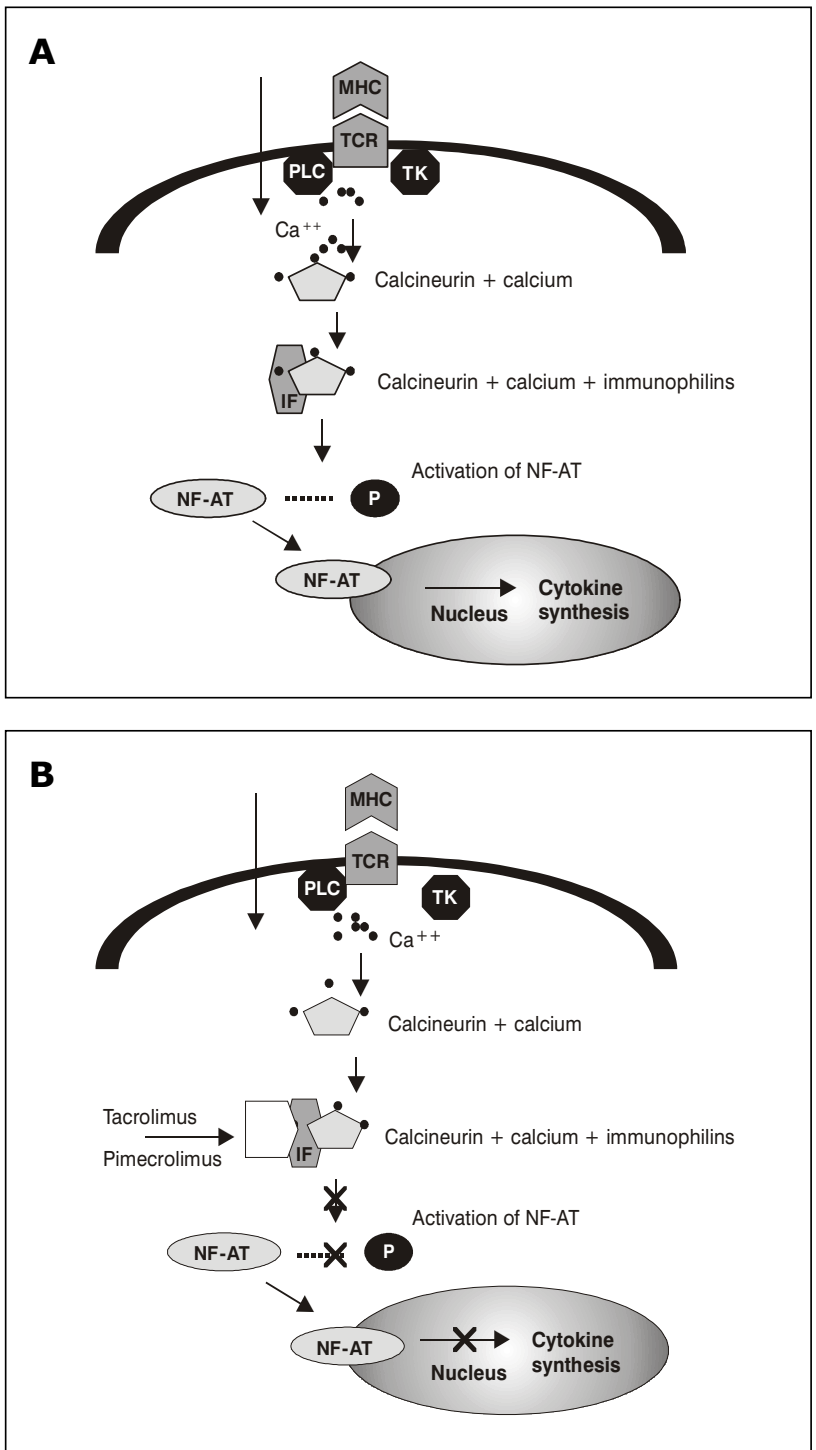

IF = immunophilin; NF-AT = transcription factor; $\mathrm{P}=$ phosphorus; $\mathrm{PLC}=$ phospholipase $\mathrm{C} ; \mathrm{TK}=$ tyrosine kinase.

Figure 1 - A) Activity of NF-AT transcription factor via calcineurin; B) Activity of calcineurin inhibitors 
hygroscopicus. Since 1992, when the first studies on pimecrolimus were carried out, researchers have observed its ability to control the inflammatory process in the skin in different dermatologic diseases of an immunological nature. ${ }^{7}$

Its mechanism of action, similarly to other immunomodulating macrolides, is related to calcineurin inhibition via macrophilin-12, the main immunophilin present in human cytosol. ${ }^{8}$ Experimental and in vitro studies have demonstrated that pimecrolimus has high affinity for macrophilin-12 and presents the following actions: $6,9,10$

- inhibition of Th1-like IL synthesis, such as IL-2 and interferon gamma;

- inhibition of Th2-like IL synthesis, such as IL-4 and IL-10.

Experimental studies assessing the transcription of lymphokine-producing genes showed that pimecrolimus is able to inhibit the transcription of those involved with the synthesis of tumor necrosis factor in lymphocytes, but it did not present any action on dendritic cells. Also, pimecrolimus reduces the histamine and tryptase release of mast cells dependent on the high-affinity IgE receptor.

It is important to stress that those actions should be restricted to the skin, so that there is no impairment of the immune function as a whole. Several studies have assessed absorption of pimecrolimus, including its application on large body surfaces (up to $92 \%$ of the affected area) and use in moderate skin lesions with high absorption rate. Due to molecule properties that provide a lipophilic nature to pimecrolimus, its blood levels were persistently low, even when used in extensive areas for more than 1 year. In a study using pimecrolimus for 3 months in adults, serum levels below $1.4 \mu \mathrm{g} / \mathrm{mL}$ were found in all patients, and $78 \%$ had levels below the method detection limit. Another study assessing patients for a 1-year period found that $98 \%$ of measurements were below the method detection limit. A study assessing the absorption kinetic of pimecrolimus in 10 children aged between $1-4$ years revealed that $63 \%$ of collected samples did not find pimecrolimus in blood circulation. Levels ranged between $0.5-1.8 \mu \mathrm{g} / \mathrm{mL}$ in the cases in which concentration of pimecrolimus was found. There are no established immunosuppression levels for pimecrolimus, but it is known that levels between $5-15 \mathrm{ng} / \mathrm{mL}$ of tacrolimus are considered immunosuppressive and are recommended for transplanted patients. ${ }^{11-15}$

\section{Tacrolimus}

Tacrolimus was discovered in 1987 by Japanese researchers who found the production of a macrolide with immunosuppressive properties by a bacterium called Streptomyces tsukubaensis, hence the name tacrolimus (tsukuba macrolide immunosuppressant). Although being initially synthesized for systemic use to prevent rejection of solid organ transplantation, a topical formulation was developed with the aim of obtaining local immunomodulating benefits without developing systemic adverse effects. As a consequence, its use is now approved for controlling allergic eczematous reactions. 6

Tacrolimus penetrates the membrane of cells involved in $A D$ inflammation. By binding to calmodulin, it inhibits the synthesis of calcineurin in lymphocytes, dendritic cells, basophils and eosinophils. The resulting immunological actions were observed in experimental and in vitro studies, revealing:

- reduction in transcription and synthesis of IL-2, IL-3, IL-4, IL-5, GM-CSF (granulocyte-macrophage colonystimulating factor) in T lymphocytes, tumor necrosis factor and interferon gamma; 16,17

- reduction in dendritic cells, which are antigen-presenting cells and help to trigger the allergic inflammatory response; $16-18$

- reduction in synthesis of mast cells and basophil mediators produced by calcineurin; ${ }^{19}$

- reduction in histamine release by peripheral basophils (in vitro studies); ${ }^{19}$

- reduction in substance $P$ levels and neuronal growth factor produced by keratinocytes, which helps reduce the pruritus. 20

Such action provides control of the inflammatory process, since $T$ lymphocytes are less activated and dendritic cells express a lower amount of high-affinity IgE receptors. However, IDEC inhibition is more pronounced compared with Langerhans cells.

With regard to the staphylococcus, which colonizes $90 \%$ of the skin in patients with AD and is a major trigger of inflammation, studies have demonstrated reduction in this bacteria population in patients treated with tacrolimus. ${ }^{21}$ In vitro studies have showed that tacrolimus has fungicidal activity against Malassezia furfur, whose allergens are related to worsening of $A D$, especially in the head and neck. ${ }^{6}$

The need of understanding the kinetics of tacrolimus applied to the skin has led to the development of a series of studies to assess its absorption and blood levels. The studies assessing tacrolimus blood concentrations have demonstrated that these levels are low, but vary according to the severity of AD. The highest serum level was not more than $3 \%$ in the concentration of patients using systemic tacrolimus. A study assessing adults and children using tacrolimus for 12 weeks showed that $80 \%$ of the samples assessed in the adult population revealed 
undetectable levels of tacrolimus; such value increased to $90 \%$ in children, and was $100 \%$ in children assessed in another study. Higher serum levels occurred more significantly in patients with larger affected areas, more severe lesions, higher amounts of drugs or in Africandescendant patients. These studies showed that there are still no significant differences between the adverse effects reported in the populations and differences in detected serum levels. 22,23

\section{Efficacy of calcineurin inhibitors}

At the same time experimental and in vitro studies confirmed the anti-inflammatory effects of those products, their activity started being assessed, when topically applied in patients with inflammatory dermatologic diseases, particularly AD. The products developed for topical assessment have acquired the following characteristics:

- pimecrolimus: $1 \%$ strength cream;

- tacrolimus: ointment available in two strengths $(0.03 \%$ and $0.1 \%$ ).

Both formulations should be applied twice daily.

To determine the efficacy of pimecrolimus and tacrolimus, several parameters have been used: physician's evaluation, patient's evaluation, reduction in pruritus and reduction in affected area. The application of severity scores is a widely used method in $A D$; for that reason, it is important to be familiar with such instruments. Scores generally assess the affected area and lesion intensity. The most used scores are: eczema and severity index (EASI) and severity scoring of atopic dermatitis (SCORAD). The latter involves subjective aspects related to pruritus intensity and sleep impairment. 24,25

Both drugs have proved to be efficacious to control AD lesions, compared with a placebo vehicle. With regard to pimecrolimus, studies have assessed its efficacy in adults, children and infants. The best results were observed when $1 \%$ pimecrolimus was applied twice daily, which shows its superiority over the control group that was given the vehicle alone. Studies of varying design have been carried out, even comparing the outcome of lesions in a single patient undergoing different treatments for each lesion. Other studies have assessed reduction in the affected area, global medical impression, severity scores and need of rescue medication with topical corticotherapy. The efficacy of pimecrolimus has been determined in children aged between 2-17 years. The mean time of use in doubleblind, placebo-controlled studies was initially 6 weeks. The patients later used that drug in a 20-week open study to assess the results. Most patients presented mild to moderate $A D$ and the results indicated reduction in lesion intensity and pruritus and, in the long term, reduction in number and intensity of new attacks. Studies in infants aged between 3-23 months revealed the same benefits found in older children. ${ }^{26-28}$

With regard to tacrolimus, initial 12-week studies demonstrated efficacy in adults and children aged between 2-15 years. The patients were advised to use the drug twice daily for up to 1 week after improvement in condition or 12 weeks at the most. Paller et al. applied tacrolimus twice daily. The results were assessed according to medical impression and affected area, showing significant reduction in the condition of patients who used tacrolimus compared with the vehicle. 22 The findings by Hanifin et al. were similar. These authors studied 632 adults and used the EASI severity scoring system to assess the results, which were also favorable to tacrolimus. ${ }^{29}$ Calcineurin inhibitors are characterized by their particular efficacy on the neck and face; the latter is a risk region for using topical corticotherapy. 30

The studies using long-term tacrolimus assessed its use for periods ranging from 6 months to 2 years. The results obtained demonstrated significant differences regarding reduction in affected area and severity scores. There was no loss of efficacy over time. ${ }^{31,32}$

When compared with corticotherapy, $0.03 \%$ tacrolimus is equivalent to low-potency topical corticosteroids; $0.1 \%$ tacrolimus has efficacy comparable to moderate-potency corticosteroids. Long-term studies have a higher dropout rate in patients using corticotherapy than in those using tacrolimus. 33,34

The treatment with calcineurin inhibitors, resulting in reduction in the inflammatory infiltrate, may influence the progress to other allergic diseases. After cohort studies have been carried out, it is known today that AD in certain patients is a major risk factor for the development of respiratory allergies; it is speculated that controlling $A D$ could contribute to reducing the prevalence of asthma and rhinitis. However, there are no completed studies stating that the chronic use of pimecrolimus and tacrolimus interferes with the frequency of respiratory allergies. ${ }^{35}$

A better understanding of their action profile was possible after their efficacy was demonstrated by several studies. In Brazil, pimecrolimus is indicated to patients older than 3 months who have mild to moderate AD and in cases of contact dermatitis. ${ }^{36}$ It should be applied twice daily as soon as the lesion is detected. Duration of treatment depends on the physician's clinical assessment. Its determination as a first or second choice drug for the treatment of $A D$ compared with topical corticotherapy is still unclear, and there is no consensus regarding this issue. In the USA, where pimecrolimus may be used in patients older than 2 years, its use is recommended as second choice drug in patients who have already used corticosteroids and had unfavorable results, adverse effects or contraindication to this drug. 
In Brazil, $0.03 \%$ tacrolimus is recommended to pediatric patients older than 2 years, and $0.1 \%$ tacrolimus has been approved for adults, similarly to the recommendation in the USA. Tacrolimus can be used in any severity level of AD. 37

The efficacy of calcineurin inhibitors in other dermatologic diseases of inflammatory nature has been studied, especially in seborrheic eczema, lupus erythematosus, psoriasis and vitiligo. However, larger series of patients need to be assessed before the real benefits of this treatment in those specific diseases can be determined. 6,26

\section{Safety \\ Local adverse effects}

Several studies show that calcineurin inhibitors are generally well tolerated. The main adverse effect was the sensation of pruritus and burning. With regard to pimecrolimus, those adverse effects have been assessed in all age groups, with frequency ranging from 1.5$25 \% .6,26$ In some reports, burning and pruritus accounted for the discontinuation of pimecrolimus. With regard to tacrolimus, a large number of studies have been performed and results are sometimes disagreeing, but it can be concluded that burning and pruritus are the main local adverse effects, and that they are more frequent in $0.1 \%$ tacrolimus. Such burning sensation usually starts soon after application of the drug, lasts for 1 hour and tends to reduce its intensity by the end of the first week of treatment. The frequency of pruritus at the application site was not always higher than the findings observed in patients who were only given the vehicle. Nevertheless, compared with corticotherapy, burning and pruritus were more frequently observed in patients using tacrolimus. 26-34

One of the concerns related to local adverse effects is to detect whether there is increase in the number of skin infectious processes after those drugs start being used. It is important to remember that the skin of patients with $A D$, a disease in which pimecrolimus and tacrolimus have been more widely studied, has immunologic changes that facilitate the increase in infections by staphylococcus and virus, especially herpes simplex and molluscum contagiosum. Reports assessing the efficacy of calcineurin inhibitors were always careful to record adverse effects related to their application. Similar incidences to patients who were only given the vehicle were observed. Open studies involving the application of tacrolimus for 1 year reported herpetic eczema and dermatophytoses. Folliculitis, however, was more frequently observed in patients using placebo when compared with those using tacrolimus. With regard to the staphylococcus infection, there was a reduction in the number of infectious processes throughout the study after a prolonged use of those drugs. ${ }^{26-34}$
A critical assessment of the results is now needed. AD leads to a series of immunological changes, including impairment of local cellular immunity, which mainly facilitates viral processes. Although the results assure safety in using pimecrolimus and tacrolimus, it is necessary to observe the patient's individual progress, whether there was previous history of skin infections or whether the clinical condition worsened after using the drugs. The treatment benefits can thus be enjoyed without the risk of local adverse effects.

One of the differentials in the treatment with calcineurin inhibitors is the possibility of using the drug for long periods without triggering local side effects associated with corticotherapy. The effects caused by inadequately applied corticotherapy are already well known. Presence of atrophy, striae, telangiectasias, vascular fragility, purpura, acneiform eruption and hypopigmentation are among the adverse effects associated with the application of topical corticotherapy. These may be due to individual characteristics of the patient's skin or to drug misuse, considering potency, application site and time. The studies on calcineurin inhibitors have not demonstrated such effects, including long-term studies.

\section{Systemic effects}

The use of topical tacrolimus or pimecrolimus did not lead to systemic adverse effects. There were no changes in renal or liver function, neither any type of impairment. This is probably due to previously discussed aspects regarding low or undetectable serum levels of those drugs. One aspect stressed in the literature is the concern for preserving the immune function, especially related to vaccine response. 38

There are two reports on tacrolimus. The first one, published in 2005, was an open study in 23 children with $A D$ who initially underwent a 7-week treatment with $0.03 \%$ tacrolimus. During the third week, the children were vaccinated against pneumococcus using the 23valent pneumococcal polysaccharide vaccine. After 4 weeks, the patients were assessed considering the immune system, which included lymphocyte cell count, CD4/CD8 ratio, immunoglobulin levels, vaccine response to previously administered vaccines (tetanus and hemophil) and pneumococcal vaccine response. The results did not show any changes in all the assessed patients, and the vaccine response against pneumococcus was considered efficacious. 39

The second study, which has been recently published, assessed the genesis of antibodies after stimulation with meningitis $C$ conjugate vaccine, this time comparing three groups: patients using tacrolimus, patients using hydrocortisone and normal controls. The authors found adequate levels in response efficacy of antibodies against $\mathrm{C}$ meningococcus $\mathrm{C}$ in the three groups. ${ }^{40}$ 
A report assessed the levels of antibodies against measles, tetanus, rubella and diphtheria in 91 patients using pimecrolimus for 1 (15 patients) or 2 years (76 patients). The levels obtained were compared to standards predicted for age group and disease. There were no significant changes in the levels of antibodies against the antigens under investigation, compared with population controls. 38

The possibility of developing neoplasias related to the use of pimecrolimus and tacrolimus deserves careful assessment. Tacrolimus and pimecrolimus, which were launched in the American market in 1999, received a warning by the Food and Drug Administration (FDA) in March 2005.41 Such warning was issued based on the description of two facts: occurrence of neoplasia in animals in experimental studies on those drugs and isolated reports of neoplasia in adults and children. Although the FDA acknowledges that there is no possibility of a direct association of the drug and the occurrence of neoplasia, it states that a correlation can only be established after the drug has been used for a long period. The FDA did not recommend suspension of the drug, but suggested that physicians should warn patients of the possible risks involved. Throughout 2005, American allergy and dermatology societies held multidisciplinary meetings and published a document evaluating FDA's statement, stressing that the data used to reach that conclusion were insufficient. The group of experts concluded that the doses used in experimental studies were much higher than those used in daily practice and underlined the fragility of case reports to establish a causal relationship. It was then stated that skin neoplasia might have been wrongly considered allergic dermatitis; therefore, calcineurin inhibitors were inappropriately used. It was also recommended that the patients who have skin lesions with increased risk of developing neoplasia, such as xeroderma pigmentosum, should not use the drug. Until a conclusive statement can be made, those societies have created a document to be given to physicians, so that they can guide patients concerning the use of the drug; however, they emphasize, including in indexed publications, that so far there has been no evidence for the warning issued by the FDA. $42-45$

Once again, it is necessary to ponder and critically and cautiously assess all facts, so that the most adequate statement can be issued, particularly focusing on the patient's well being. To date, pimecrolimus and tacrolimus have been used by more than 2 million patients in the USA. Reports by the pharmacovigilance system do not indicate increased prevalence of neoplasia compared with the population that does not use those drugs. Studies published in judicious journals stress that current evidence, pharmacological characteristics and vigilance after the drug was launched do not indicate higher risks of developing neoplasia. 45

Calcineurin inhibitors are still used, but some cares should be taken by following the FDA guidelines. It is important to use the drug following the directions for taking it, which reflect the indications approved by regulating agencies. In Brazil, calcineurin inhibitors are approved for the treatment of $A D$ and contact dermatitis. It is important to advise patients as to the need of solar protection, a measure that should have been widely recommended. The use of calcineurin inhibitors should be intermittent, i.e., even if it is used over a long time, it should only be applied during exacerbation and up to 1 week after improvement. The treatment should be recommended when the corticotherapy is inefficient or when the lesion site is likely to lead to adverse effects related to the corticotherapy, such as, for example, in eyelids. All those measures provide therapeutic advantages and risk minimization. These events reinforce the need of having a physician prescribing the drug and maintaining the patient's follow-up throughout the whole treatment and after it has been completed. This generates more knowledge on the therapy and allows patients to enjoy the best benefits of the treatment as safely as possible.

Calcineurin inhibitors are a new therapeutic class for the management of allergic dermatitis. They have been widely studied. Thousands of patients of varying age groups participated in clinical studies leading to the conclusion that those drugs help control AD, by improving attacks, minimizing the number of exacerbations throughout 1 year and reducing corticotherapy courses. Calcineurin inhibitors may contribute to reduction in bacterial and fungal colonization. They are well tolerated drugs during application, and the main adverse effects are related to pruritus and burning. Serum levels after use are very low or undetectable, and the studies assessing the immune function did not show any impairment of this system. Concern has been raised by the FDA as to the potential risk of developing neoplasia, but current studies do not allow the establishment of a direct correlation. Such drugs should always be prescribed by physicians, for the diseases to which they have been recommended and licensed.

\section{References}

1. Leung DY, Nicklas RA, Li JT, Bernstein IL, Blessing-Moore J, Boguniewicz M, et al. Disease management of atopic dermatitis: an updated practice parameter. Joint Task Force on Practice Parameters. Ann Allergy Asthma Immunol. 2004;93:S1-21.

2. Leung DY. Immunopathogenesis of atopic dermatitis. Immunol Allergy Clin North Am. 2002;22:73-90.

3. Leung DY, Boguniewicz M, Howell MD, Nomura I, Hamid QA. New insights into atopic dermatitis. J Clin Invest. 2004; 113:651-7. 
4. Novak $\mathrm{N}$, Bieber $\mathrm{T}$. The role of dendritic cell subtypes in the pathophysiology of atopic dermatitis. J Am Acad Dermatol. 2005;53:S171-6.

5. Militello G, Jacob SE, Crawford GH. Allergic contact dermatitis in children. Curr Opin Pediatr. 2006;18:385-90.

6. Simpson D, Noble S. Tacrolimus ointment: a review of its use in atopic dermatitis and its clinical potential in other inflammatory skin conditions. Drugs. 2005;65:827-58.

7. Meingasser JG, Stutz A. Immunosuppressive macrolides: a novel class of topical agents for treatment of skin disease. J Invest Dermatol. 1992;98:851-5.

8. Kay JE. Structure-function relationships in the FK506-binding protein (FKBP) family of peptidylprolyl cis-trans isomerases. Biochem J. 1996;314:361-85.

9. Meingassner JG, Grassberger M, Fahrngruber $H$, Moore HD, Schuurman $\mathrm{H}$, Stutz A. A novel anti-inflammatory drug, SDZ ASM 981, for the topical and oral treatment of skin diseases: in vivo pharmacology. Br J Dermatol. 1997;137:568-76.

10. Zuberbier T, Chong SU, Grunow K, Guhl S, Welker P, Grassberger $M$, et al. The ascomycin macrolactam pimecrolimus (Elidel, SDZ ASM 981) is a potent inhibitor of mediator release from human dermal mast cells and peripheral blood basophils. J Allergy Clin Immunol. 2001;108:275-80.

11. Roos TC, Geuer S, Roos S, Brost H Recent Advances in Treatment Strategies for Atopic Dermatitis. Drugs. 2004;64: 2639-66.

12. Concentrations of pimecrolimus (Elidel, SDZ ASM 981) after topical treatment of adults with atopic dermatitis. J Eur Acad Dermatol Venereol. 2001;15:S109.

13. Van Leent EJ, Ebelin ME, Burtin P, Dorobek B, Spuls PI, Bos JD. Low systemic exposure after repeated topical application of pimecrolimus (Elidel, SDZ ASM 981) in patients with atopic dermatitis. Dermatology. 2002;204:63-8.

14. Harper J, Green A, Scott G, Gruendl E, Dorobek B, Cardno M, et al. First experience of topical SDZ ASM 981 in children with atopic dermatitis. Br J Dermatol. 2001;144:781-7.

15. Allen BR, Lakhanpaul M, Morris A, Lateo S, Davies T, Scott G, et al. Systemic exposure, tolerability, and efficacy of pimecrolimus cream $1 \%$ in atopic dermatitis patients. Arch Dis Child. 2003;88:969-73.

16. Thomson AW, Bonham CA, Zeevi A. Mode of action of tacrolimus (FK506): molecular and cellular mechanisms. Ther Drug Monit. 1995; 17:584-91.

17. Reynolds NJ, Al-Daraji WI. Calcineurin inhibitors and sirolimus: mechanisms of action and applications in dermatology. Clin Exp Dermatol. 2002;27:555-61.

18. Wollenberg A, Sharma S, von Bubnoff D, Geiger E, Heberstok J, Bieber T. Topical tacrolimus (FK506) leads to profound phenotypical and functional alterations of epidermal antigenpresenting dendritic cells in atopic dermatitis. J Allergy Clin Immunol. 2001;107:519-25.

19. de Paulis A, Cirillo R, Cicarelli A, Columbo M, Marone G. Antiinflammatory effect of FK 506 on human basophils. Transplant Proc. $1991 ; 23: 2905-6$.

20. Toyoda M. Antipruritic mechanism of tacrolimus ointment for atopic dermatitis: consideration of dermatoneuronal factors. Clinical Dermatology. 2003;57:45-50.

21. Remitz A, Kyllonen H, Granlund H, Reitamo S. Tacrolimus ointment reduces staphylococcal colonization of atopic dermatitis lesions. J Allergy Clin Immunol. 2001;107:196-7.

22. Paller A, Eichenfield LF, Leung DY, Stewart D, Appell M. A 12week study of tacrolimus ointment for the treatment of atopic dermatitis in pediatric patients. J Am Acad Dermatol. 2001;44:S47-57.

23. Soter NA, Fleischer AB Jr., Webster GF, Monroe E, Lawrence I. Tacrolimus ointment for the treatment of atopic dermatitis in adult patients: part II, safety. J Am Acad Dermatol. 2001;44: S39-46.

24. Hanifin JM, Thurston M, Omoto M, Cherill R, Tofte SJ, Graeber $M$. The eczema area and severity index (EASI): assessment of reliability in atopic dermatitis. EASI Evaluator Group. Exp Dermatol. 2001;10:11-8.

25. Severity scoring of atopic dermatitis: the SCORAD index. Consensus Report of the European Task Force on Atopic Dermatitis. Dermatology. 1993;186:23-31.

26. Wellington $\mathrm{K}$, Jarvis $\mathrm{B}$. Topical pimecrolimus: a review of its clinical potential in the management of atopic dermatitis. Drugs. 2002;62:817-40
27. Van Leent EJ, Graber M, Thurston M, Wagenaar A, Spuls PI, Bos JD. Effectiveness of the ascomycin macrolactam SDZ ASM 981 in the topical treatment of atopic dermatitis. Arch Dermatol. 1998; 134:805-9.

28. Grassberger $M$, Baumruker $T$, Enz A, Hiestand $P$, Hultsch $T$, Kalthoff $F$, et al. A novel anti-inflammatory drug, SDZ ASM 981, for the treatment of skin diseases: in vitro pharmacology. $\mathrm{Br}$ J Dermatol. 1999;141:264-73.

29. Hanifin JM, Ling MR, Langley R, Breneman D, Rafal E. Tacrolimus ointment for the treatment of atopic dermatitis in adult patients: part I, efficacy. J Am Acad Dermatol. 2001;44:S28-38.

30. FK 506 Ointment Study Group. Phase III comparative study of FK 506 ointment vs. alclometasone dipropionate ointment in atopic dermatitis (face and neck). Acta Dermatol (Kyoto). 1997; 92:277-88.

31. Reitamo S, Wollenberg A, Schopf E, Perrot JL, Marks R, Ruzicka $T$, et al. Safety and efficacy of 1 year of tacrolimus ointment monotherapy in adults with atopic dermatitis. Arch Dermatol. 2000;136:999-1006.

32. Kang S, Lucky AW, Pariser D, Lawrence I, Hanifin JM. Long-term safety and efficacy of tacrolimus ointment for the treatment of atopic dermatitis in children. J Am Acad Dermatol. 2001;44: S58-64.

33. Reitamo S, Ortonne JP, Sand C, Cambazard F, Bieber T, FolsterHolst R, et al. A multicenter, randomized, double-blind, controlled study of long-term treatment with $0.1 \%$ tacrolimus ointment in adults with moderate to severe atopic dermatitis. Br J Dermatol. 2005; 152:1282-9.

34. Reitamo S, Rustin M, Ruzicka T, Cambazard F, Kalimo K, Friedmann PS, et al. Efficacy and safety of tacrolimus ointment compared with that of hydrocortisone butyrate ointment in adult patients with atopic dermatitis. J Allergy Clin Immunol. 2002; 109:547-55.

35. Boguniewicz M. Update on atopic dermatitis: insights into pathogenesis and new treatment paradigms. Allergy Asthma Proc. 2004;25:279-82.

36. Bula do Pimecolimo. http://www.elidel.info/BZ/PT/protected/ Elidel_BULA.doc Access: 20/08/2006.

37. Bula do Tacrolimo. http://www.roche.com.br/Products/ protopic PT.htm?setcategory=1 Access: 20/08/2006.

38. Hofman T, Cranswick N, Kuna P, Boznanski A, Latos T, Gold M, et al. Tacrolimus ointment does not affect the immediate response to vaccination, the generation of immune memory, or humoral and cell-mediated immunity in children. Arch Dis Child. 2006; [Epub ahead of print].

39. Stiehm ER, Roberts RL, Kaplan MS, Corren J, Jaracz E, Rico MJ. Pneumococcal seroconversion after vaccination for children with atopic dermatitis treated with tacrolimus ointment. J Am Acad Dermatol. 2005;53:S206-13.

40. Papp KA, Breuer K, Meurer M, Ortonne JP, Potter PC, de Prost Y, et al. Long-term treatment of atopic dermatitis with pimecrolimus cream $1 \%$ in infants does not interfere with the development of protective antibodies after vaccination. J Am Acad Dermatol. 2005; 52:247-53

41. Food and Drug Administration. Warning concerning long-term safety of topical calcineurin. http://www.fda.gov/cder/foi/label/ 2006/021302s011lbl.pdf. Access: 21/08/2006.

42. American Academy of Dermatology. American Academy of Dermatology issues statement in response to FDA decision related to two eczema medications. http://www.aad.org/public/ News/NewsReleases/fda_decision.htm. Access: 21/08/2006.

43. http://www.aad.org/NR/rdonlyres/752ED70B-1424-4985A752- 50835CABDE85/0/Patient_info_10_20.pdf. Access: 21/ $08 / 2006$.

44. Ormerod AD. Topical tacrolimus and pimecrolimus and the risk of cancer: how much cause for concern? Br J Dermatol. 2005; 153: 701-5.

45. Spergel JM, Leung DY. Safety of topical calcineurin inhibitors in atopic dermatitis: evaluation of the evidence. Curr Allergy Asthma Rep. 2006;6:270-4.

Correspondence:

Ana Paula B. Moschione Castro

Rua Pedro Pomponazzi, 890/21 - Vila Mariana

CEP 04115-050 - São Paulo, SP - Brazil

E-mail: moschione@uol.com.br 